\title{
Did globular clusters contribute to the stellar population of the Galactic halo?
}

\author{
Corinne Charbonnel ${ }^{1,2}$ and Martin Krause ${ }^{3,4}$ \\ ${ }^{1}$ Department of Astronomy, University of Geneva \\ 1290 Versoix - Switzerland \\ email: Corinne.Charbonnel@unige.ch \\ ${ }^{2}$ IRAP CNRS UMR 5277, Université de Toulouse III \\ 31400 Toulouse, France \\ ${ }^{3}$ Universitäts-Sternwarte München, Ludwig-Maximilians-Universität \\ Scheinerstr. 1, 81679 München, Germany \\ email: krause@mpe.mpg.de \\ ${ }^{4}$ Max Planck Institute for extraterrestrial Physics, \\ PO Box 1312, Giessenbachstr., 85741 Garching, Germany
}

\begin{abstract}
The origin of Galactic halo stars and the contribution of globular clusters (GC) to this stellar population have long been (and still are) debated. The discovery of multiple stellar populations with peculiar chemical properties in GCs both in the Milky Way and in Local Group galaxies recently brought a renewal on these questions. Indeed most of the scenarios that compete to reproduce the present-day GC characteristics call for fast expulsion of both gas and low-mass stars from these clusters in their early infancy. In this framework, the initial masses of GCs could have been 8 to 25 times higher than their present-day stellar mass, and they could have contributed to 5 to $20 \%$ of the low-mass stars in the Galactic halo. Here we revisit these conclusions, which are in tension with observations of dwarf galaxies and of young massive star clusters in the Local Group. We come back in particular on the paradigm of gas expulsion from massive star clusters, and propose an alternative interpretation of the GC abundance properties. We conclude by proposing a major revision of the current concepts regarding the role massive star clusters play in the assembly of galactic haloes.
\end{abstract}

Keywords. globular clusters: general

\section{Introduction}

Globular clusters (GC) have long been considered as the perfect archetype of single stellar populations of coeval stars born with the same chemical composition. Indeed and although important differences in metallicity from cluster to cluster were long recognized, no significant star-to-star $[\mathrm{Fe} / \mathrm{H}]$ abundance variations are found within individual clusters except the most massive ones (e.g., Omega Cen, M54, M22, NGC 1851, NGC 5286; see e.g. Simmerer et al. 2013, Marino et al. 2015 and references therein).

A major paradigm shift has occurred in the domain since the early 2000's, thanks to very high resolution spectroscopy with 8-10m class telescopes, together with highprecision photometry of Galactic GCs especially performed with HST (also ground based photometry is showing the same effects of MP, eg with Stromgren filters, or the various U filters). Those challenging and revolutionary studies have revealed that individual GCs do actually host multiple stellar populations. Those manifest themselves through their different chemical properties, in particular the well-documented O-Na anticorrelation that appears to be ubiquitous in GCs more massive than a few $10^{4} \mathrm{M}_{\odot}$ (e.g. Carretta et al. 2009a, Carretta et al. 2010, Lind et al. 2009, Gratton et al. 2012). These peculiarities 
are associated to exquisite photometric complexity, with the appearance of multimodal sequences in different regions of the GC color-magnitude diagrams (e.g. Piotto et al. 2002, Milone et al. 2015). These properties have not been found yet in open clusters (Bragaglia et al. 2014). However, spectroscopic searches of field halo giant stars with chemical properties similar to that of GCs have opened the possibility that a large fraction of the halo population was originally formed in globular clusters (Martell et al. 2011). To improve the statistics, these studies are based on $\mathrm{CN}$ and $\mathrm{CH}$ bandstrength behavior, which is easily determined, but which might not reflect the initial composition of the stars as $\mathrm{C}$ and $\mathrm{N}$ are subject to evolution variations induced by internal mixing processes within the red giant stars themselves (e.g. Charbonnel \& Zahn 2007a, Charbonnel \& Lagarde 2010, Lagarde et al. 2012). Such intrinsic variations should be accounted for when estimating the actual number of peculiar stars that have really escaped from GCs. This is extremely important as the percentage of field halo stars with chemical properties similar to those observed in GCs does put, in principle, tight constraints on the actual contribution of GCs to the halo stellar populations.

\section{Self-enrichment scenarii and the mass budget problem}

Different scenarii were built to explain GCs chemical and photometric patterns. Most call for "self-enrichment" of GCs during their infancy, implying the formation of at least two stellar populations in all GCs. In this framework, first population (1P) stars are thought to be born with the proto-cluster original composition (i.e., that of contemporary field halo stars), while second population (2P) stars formed from original gas polluted to various degrees by hydrogen-burning processed material ejected by more massive, short-lived, 1P GC stars. The nature of the "polluters" is strongly debated, with the most commonly-invoked ones being the so-called Fast Rotating Massive Stars (FRMS; masses higher than $\sim 20 \mathrm{M}_{\odot}$; Decressin et al. 2007a, Decressin et al. 2007b, Krause et al. 2013) and massive AGB stars (masses $\sim 6-6.5 \mathrm{M}_{\odot}$; e.g. Ventura et al. 2001, Ventura et al. 2013, D'Ercole et al. 2010). Variants and combinations of these objects have been proposed as possible solutions, as well as supermassive stars $\left(\sim 10^{4} \mathrm{M}_{\odot}\right.$ stars; Denissenkov \& Hartwick 2014).

When considering a classical stellar IMF for the polluters, both the FRMS and the AGB scenarii face the so-called "mass-budget" problem. Indeed, the ratio between $1 \mathrm{P}$ and $2 \mathrm{P}$ stars is predicted to be of the order of ten to one, even when assuming that the maximum amount of their $\mathrm{H}$-processed ejecta is entirely recycled into the second stellar population after a 50-50\% dilution with pristine gas. To make things even worse, this 10-1 ratio is obtained when assuming that the $2 \mathrm{P}$ was composed of low-mass stars only. However, the percentage of $2 \mathrm{P}$ GC stars observed today in GCs is $\sim 70 \%$, with only slight variations from cluster to cluster (e.g. Prantzos \& Charbonnel 2006, Carretta 2013).

\section{Gas and star expulsion from GCs in their infancy?}

Drastic gas expulsion has been identified as a way to enhance the ratio of $2 \mathrm{P}$ to $1 \mathrm{P}$ stars (e.g. Prantzos \& Charbonnel 2006, D'Ercole et al. 2010, Schaerer \& Charbonnel 2011). If the $2 \mathrm{P}$ was formed in the immediate surrounding of the polluters at the center of a mass-segregated cluster, the less tightly bound $1 \mathrm{P}$ would have been lost preferentially as a result of a strong change in the GC potential well induced by gas expulsion (Decressin et al. 2010). In order to overcome the mass budget issue within this standard IMF framework, the initial masses of GCs must be $\sim 8-10$ times (or up to 25 times, if $2 \mathrm{P}$ stars also escape from GCs, as suggested by the "CN-CH surveys", see $\S 1$ ) larger than 
the present-day stellar mass. Therefore, the present-day Galactic GC population would then have contributed to approximately 5 to $8 \%$ (10 to $20 \%)$ of the low-mass stars in the Galactic halo (Schaerer \& Charbonnel 2011, Martell et al. 2011).

For this to work, N-body simulations require explosive gas expulsion to unbind $\geqslant 95$ $\%$ of $1 \mathrm{P}$ stars; more precisely, it must happen on the crossing timescale of the cluster (Decressin et al. 2010, Khalaj \& Baumgardt 2015). According to gas expulsion models happening via the formation of a superbubble, with kinematics described by a thin-shell model (Krause et al. 2012, Krause et al. 2013), stellar winds and supernovae explosions fail to produce sufficient power for this process to work. With these sources of energy, the superbubbles are destroyed by the Rayleigh-Taylor instability before they reach escape speed for all but perhaps the least massive and most extended clusters. Rather, energy released by the coherent onset of accretion onto the stellar remnants of $1 \mathrm{P}$ massive stars (neutron stars and black holes) might plausibly accomplish the task (Krause et al. 2012). However, this fast episode of gas and stars removal would happen at the end of the SNe phase, when turbulence decreases within the ISM and all massive stars have turned into such dark remnants, i.e., typically $\sim 35 \mathrm{Myr}$ after cluster formation.

\section{Gas expulsion from Young Massive Star Clusters}

\subsection{Observational constraints}

These conclusions are in tension with observations of young massive star clusters (YMC) in the Local Group and in dwarf galaxies, that can potentially test the conditions for gas expulsion. In particular, a sample of YMC with ages below $10 \mathrm{Myr}$, current masses between 8 and $50 \times 10^{5} \mathrm{M}_{\odot}$, and half-mass radii between 1.5 and $18 \mathrm{pc}$ (i.e., very similar to the properties of GC progenitors), appear to be gas free by an age of $\sim 3 \mathrm{Myr}$, and show no evidence for multiple epochs of star-formation (Bastian et al. 2014, hereafter B14). In the case GC formation at high redshift happened the same way as YMC formation today, this implies that the dark remnant scenario for fast gas removal occurs too late to explain the disappearance of the gas at such an early stage.

\subsection{Grid of thin shell models for the gas dynamics}

To solve this inconsistency and understand how such YMCs could lose their gas so early in their evolution, we have computed a tailor-made grid of thin shell models for the gas dynamics (Krause et al. 2015, hereafter K15). We considered different sources of energy production (metallicity dependent stellar winds, radiation, supernovae, winds of young pulsars, and gamma ray burst related hypernovae). We used standard assumptions for the stellar IMF (Kroupa et al. 2013) and for the coupling between the energy produced by stellar feedback and the gas (20\% efficiency). We first assumed a local star formation efficiency (SFE) of $30 \%$, which is the preferred value for self-enrichment scenarios, and then we increased this quantity in some of our models.

With this grid, we show that the success of gas expulsion is strongly and mainly dependent on the compactness $\dagger$ of a star cluster as well as on the local SFE. We find that for two out of the eight YMCs in the sample of B14 gas expulsion is possible with a SFE of $30 \%$ for both the winds and SNe energy injection cases. However, none of these processes is sufficient to expel the residual gas for six YMCs in the sample, if the SFE was $30 \%$. We find that $10^{52}$ erg hypernovae would be needed to expel the gas at this SFE

$\dagger$ We compute the compactness index with the formula $\mathrm{C}_{5} \equiv\left(\mathrm{M}_{*} / 10^{5} \mathrm{M}_{\odot}\right) /\left(\mathrm{r}_{h} / \mathrm{pc}\right)$, with the initial stellar mass $\mathrm{M}_{*}$ and half-mass radius $\mathrm{r}_{h}$. 
in one case (T352/W38220), and $10^{53}$ erg hypernovae would be required for the other four.

A possible way to remove residual gas is to assume a higher star formation efficiency. For two of the eight clusters of B14, an increase of the SFE up to $\sim 50 \%$ is sufficient to account for gas expulsion. For the four most compact YMCs however, the SFE has to be $\sim 90 \%$ to remove the remaining case. However in that case, the associated change of potential well is far too small to lead to a significant loss of stars, and the contribution of the clusters to the field population is minute compared to the standard framework for GC self-enrichment.

\section{What if YMCs happen to be the modern counterparts of GCs that formed in the early universe?}

YMC formation happening in the local and modern universe might be totally different than GC formation at high redshift. However, in the case where YMCs happen to be the modern counterparts of GCs, we should conclude that gas and star expulsion is a serious issue for the current GC self-enrichment scenarii that make classical assumptions for the SFE and for the stellar IMF of the first and second stellar populations. Rather, gas could be cleared for very high SFE, but in that case the total stellar mass would not be changed significantly enough to affect the $1 \mathrm{P}$-to-2P ratio as requested by the $\mathrm{O}-\mathrm{Na}$ anticorrelation observed in all GCs. Unfortunatly, the detection of the O-Na anticorrelation in YMCs is not yet feasible, although this would be the definitive test to assess the genetic filiation, or real relashionship, between YMCs and GCs. Indeed and as can be seen in Fig. 1, the distinction between star clusters showing or not the Na-O anticorrelation is clear in the $\mathrm{C}_{5-}[\mathrm{Fe} / \mathrm{H}]$ plane.

Alternative solutions have been investigated to alleviate the mass budget issue. An interesting option is to assume a flat IMF for the polluters (Smith \& Norris 1982, D'Antona \& Caloi 2004, Prantzos \& Charbonnel 2006, Schaerer \& Charbonnel 2011). Enlarging the mass domain of the polluters was also proposed, by including i.e. massive binary stars ( 9 to $20 \mathrm{M}_{\odot}$ ) as an additional source of H-burning ashes (De Mink et al. 2009), or by considering hypothetical supermassive stars $\left(\sim 10^{4} \mathrm{M}_{\odot}\right)$ as the source of abundance anomalies. Alternatively, a scenario was proposed where a single stellar generation forms, where low-mass stars with proto-planetary discs sweep up material ejected by interacting binaries or rapidly rotating massive stars (Early-Disc Accretion scenario; Bastian et al. 2013). However, all these scenarii appear to have serious drawbacks (see e.g. Bastian \& Lardo 2015).

\section{Do GCs really host $1 \mathrm{P}$ stars?}

Finally, we proposed an alternative interpretation of the observed sodium distribution, and suggested that stars with low sodium abundance that are counted as $1 \mathrm{P}$ members could actually be $2 \mathrm{P}$ stars (Charbonnel et al. 2014). For this, we computed the number ratio of $2 \mathrm{P}$ stars along the $\mathrm{Na}$ distribution following the FRMS model using tight constraints from the well-documented case of NGC 6752. We showed that the typical percentage of low-sodium stars usually classified as $1 \mathrm{P}$ stars can be reproduced by invoking only secondary star formation from material ejected by massive stars and mixed with original GC material in proportions that account for the $\mathrm{Li}-\mathrm{Na}$ anti-correlation in this cluster. In other words, GCs could be totally devoid of $1 \mathrm{P}$ low-mass stars, and all the low-mass stars we observe today could have formed out of the ejecta of $1 \mathrm{P}$ massive stars mixed with original gas in roughly 50 to 50 proportions. 


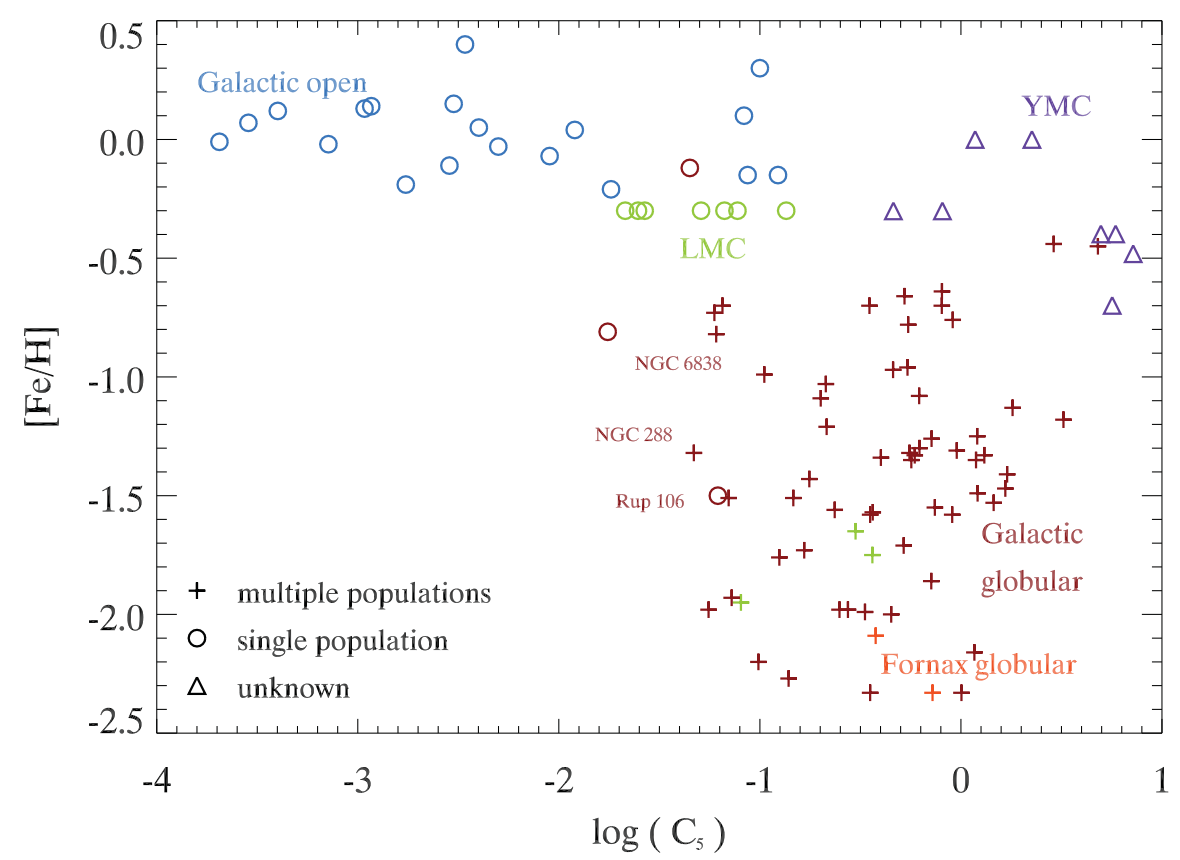

Figure 1. $[\mathrm{Fe} / \mathrm{H}]$ versus compactness index $\mathrm{C}_{5}$ for star clusters of various types and origins. Different symbols are used according to the presence or absence of the O-Na anticorrelation (plus and circles respectively; triangles are for clusters where the presence of the anticorrelation has not been assessed). Multiple populations occur at high $\mathrm{C}_{5}$ where gas expulsion becomes increasingly difficult and eventually impossible due to large binding energies. See K15 for more details and for sample references

If the absence of "true" $1 \mathrm{P}$ stars in GCs today is confirmed (this can be tested by deriving the carbon isotopic ratio in GC low-mass main sequence stars; Charbonnel et al. 2014), we should find out whether all $1 \mathrm{G}$ low-mass stars were lost from the GCs or whether they have not formed, and why. The first case seems very improbable in view of the mass budget and gas expulsion problems, which would be even more exacerbated than discussed in the present paper. Also, this would be in tension with the recent census of halo stars compared to the number of GCs in the dwarf galaxy Fornax (Larsen et al. 2014).

In the second case (no formation of $1 \mathrm{P}$ low-mass stars), the mass initially locked in $1 \mathrm{P}$ GC massive stars could have been only two to four times the present-day stellar mass (instead of 8 to 25 times, as discussed previously). This would definitively release the current tension between the different model predictions and the constraints coming from YMCs as well as from dwarf galaxies haloes. We should then understand why LMS could not form initially. Work is in progress on all these open issues.

\section{Acknowledgements}

CC acknowledges support from the Swiss National Science Foundation (FNS) for the project 200020-159543 "Multiple stellar populations in massive star clusters - Formation, evolution, dynamics, impact on galactic evolution" (PI CC). MK acknowledges support from Deutsche Forschungsgemeinschaft under DFG project number PR 569/10-1 in the context of the Priority Program 1573 Physics of the Interstellar Medium. We thank the International Space Science Institute (ISSI, Bern, CH) for welcoming the activities of ISSI Team 271 "Massive star clusters across the Hubble Time (2013 - 2016, team leader 
CC), as well as our colleagues, N.Bastian and R.Diehl, co-authors of K15 paper, and F.Primas, W.Chantereau, and Y.Wang, co-authors of C14.

\section{References}

Bastian, N. \& et al. , 2013, MNRAS, 436, 2398

Bastian, N., \& Hollyhead, K, Cabrera-Ziri, I., 2014, MNRAS, 445, 378

Bastian, N., \& Lardo, C., 2015, MNRAS, 453, 357

Bragaglia, A., et al. 2014, ApJ, 796, 68

Carretta, E., et al. 2009, A\&A 505, 139

Carretta, E., et al. 2010, A\& $A, 516, \mathrm{~A} 55$

Carretta, E. 2013, A\&A, 557, A128

Charbonnel, C. \& Lagarde, N. 2010, A\&SA, 522, A10 (C14)

Charbonnel, C. \& Zahn, J. P. 2007, A\&A (Letter), 467, L15

Charbonnel, C., Chantereau, W., Krause, M., Primas, F., \& Wang, Y. 2014, A\&A (Letter), 569, L6

D'Antona, F. \& Caloi, V. 2004, ApJ, 611, 871

Decressin, T., et al. 2007a, A\&GA, 475, 859

Decressin, T., Charbonnel, C., \& Meynet, G, 2007b, A\&A, 464, 1029

Decressin, T., Baumgardt, H., Charbonnel, C., \& Kroupa, P., A\&A, 516, A73

De Mink, S., et al. 2009, A\&A (Letters), 507, L1

Denissenkov, P. A. \& Hartwick, F. D. A.. 2014, MNRAS (Letter), 437, L21

D'Ercole, et al. 2010, MNRAS, 407, 854

Khalaj, P. \& Baumgardt,H. 2015, MNRAS, 492, 924

Gratton, R. G., Carretta, E., \& Bragaglia, A. 2012, A\&ARv, 20, 50

Krause, M. Charbonnel, C., et al. 2012, A\&A (Letter), 546, L5

Krause, M. Charbonnel, C., et al. 2013, A\&A A, 552, A121

Krause, M., Charbonnel, C., Bastian, N., \& Diehl, R. 2015, submitted to A\&A (K15)

Kroupa, P., et al. 2013, in Planets, Stars and Stellar Systems (Springer Netherlands), 115

Lagarde, N., et al. 2012, A\&A, 543, A108

Larsen, S. S., et al. 2014, A\&A, 565, A98

Lind, K., et al. 2009, A\& $A, 503,545$

Marino, A. F., et al. 2015, MNRAS, 450, 815

Martell, S. L., Molinski, J. P., Beers, T. C., \& Grebel, E. K. 2011, A\&̛A, 354, A136

Milone, A. P., Marino, A. F., Piotto, G. P., et al. 2015, ApJ, 808, 51

Piotto, G., et al. 2002, ApJ, 670, 39

Prantzos, N. \& Charbonnel, C. 2006, A\&A, 458, 135

Schaerer, D. \& Charbonnel, C. 2011, MNRAS, 413, 2297

Simmerer, J., et al. 2013, ApJ (Letters), 764, L7

Smith, G. H. \& Norris, J. 1982, ApJ, 254, 594

Ventura, P., et al. 2001, ApJ (Letter), 550, L65

Ventura, P., et al. 2013, MNRAS, 431, 3642 